Bangladesh J. Pl. Breed. Genet., 27(2): 09-15, 2014

\title{
COMBINING ABILITY FOR YIELD AND ITS COMPONENTS IN WHITE GRAIN QUALITY PROTEIN MAIZE
}

\author{
A. Ahmed, M. Amiruzzaman, S. Begum, M.M. Billah and M.M. Rohman \\ Plant Breeding Division \\ Bangladesh Agricultural Research Institute \\ Joydebpur, Gazipur 1701, Bangladesh \\ Corresponding Author: asgar.gene@gmail.com
}

\begin{abstract}
Combining ability for yield and yield components were carried out in a $8 \times 8$ diallel cross of white grain quality protein maize (QPM) to determine the general combining ability (GCA) of the parents and specific combining ability (SCA) of the crosses. Significant mean sum of squares due to GCA and SCA were observed for all the characters studied. Higher magnitude of SCA variance than GCA variance clearly indicated the predominance of non-additive gene action for all the traits. The parental lines $\mathrm{P}_{4}, \mathrm{P}_{7}$ and $\mathrm{Q}_{6}$ were found to be the best general combiner for yield components and these parents could be used as donor parents in hybridization to improve traits like days to tasseling, days to silking, plant height, ear height, ear length, ear diameter, grains per ear and 1000 grain weight by accumulation of favorable genes. The significant positive SCA along with high mean yield, the crosses $\mathrm{Q}_{5} \times \mathrm{P}_{7}, \mathrm{P}_{7} \times \mathrm{Q}_{6}$ and $\mathrm{P}_{7} \times \mathrm{Q}_{2}$ could be used for commercial variety development, after verifying their performance over locations.
\end{abstract}

Keywords: Combining ability; QPM; GCA; SCA; gene action

DOI: http://dx.doi.org/10.3329/bjpbg.v27i2.27848

\section{INTRODUCTION}

In the world, maize (Zea mays L., $2 \mathrm{n}=20$ ) ranks third important cereal crop next to wheat and rice in production. The plant is native to Central America, but its suitability to diverse environments, unmatched by any crop, makes it to expand new areas and environment continuously and thus, it explores Asia. The demand of maize increased gradually during nineties with the expansion of poultry industry and this is why, maize yields increased from an average of less than 1 ton per hectare for several decades through 1992 to more than 6 tons per hectare in 2010 by the introduction of hybrid maize varieties. Maize yields per hectare in Bangladesh exceed yields in China and Japan (Bodker, Wulff, and Thorp 2006). The increase in net income from hybrids can be estimated by assuming that farmers replace wheat with maize (Harun-Ar-Rashid et. al., 2012). In world, maize grown with a production 875 million tons (FAO, 2012), while 23 lac tons has been produced in Bangladesh occupies an area of 3.5 lac hectare (Baral, 2016). About $30 \%$ of world production is used for direct human consumption and as an industrial input, while 70\% is used as animal feed (Pavan, 2009). But in Bangladesh, the sole maize (except popcorn and adulteration in wheat flour) is used up by poultry industries. In this circumstances, white grain QPM maize can play an vital role. However, there was no such variety in Bangladesh and to meet the challenge, 
Bangladesh Agricultural Research Institute (BARI) introduce some white grain QPM maize inbreds from International Maize and What Improvement Centre (CIMMYT) and eight inbreds were selected.

To determine GCA and SCA information of white grain maize germplasm for identification of nature of genes action involved in the expression of their quantitative traits, genetic diversity evaluation, suitable parental lines selection for hybridization, heterotic pattern classification, heterosis estimation, and hybrids development, the present investigation was undertaken as the above information can be achieved only by combining ability study (Fan et al., 2002; Melani and Carena, 2005; Barata and Carena, 2006; Bello and Olaoye, 2009).

\section{MATERIALS AND METHODS}

Eight white grain QPM inbred lines $\left(\mathrm{P}_{2}, \mathrm{P}_{4}, \mathrm{P}_{6}, \mathrm{Q}_{3}, \mathrm{Q}_{5}, \mathrm{P}_{7}, \mathrm{Q}_{6}\right.$ and $\mathrm{Q}_{2}$ as parent 1, parent 2 parent 3 parent 4 parent 5 parent 7 and parent 8 , respectively) selected based on phenotypic characters were mated in a $8 \times 8$ diallel fashion without reciprocals during rabi season in 2013-2014 and their performance (28 $\mathrm{F}_{1}$ 's and their parents) were examined with four checks (BARI hybrid maize 5, BARI hybrid maize 7, BARI hybrid maize 9 and 900M) in a Alpha Lattice Design with two replications at the experimental field of BARI, Gazipur in the following rabi season 2014-2015. Notably, P and Q series inbreds were extracted from two different populations. Each and every entry was sown in a single row of $4 \mathrm{~m}$ long plot and by proper thinning one plant per hill was retained. The spacing between rows was $60 \mathrm{~cm}$ and between plants was $20 \mathrm{~cm}$.

The data of plant height $(\mathrm{cm})$, ear height $(\mathrm{cm})$, ear length $(\mathrm{cm})$, ear diameter $(\mathrm{cm})$, number of grains per ear, 1000 grain weight $(\mathrm{g})$ and yield/plant from each plot were recorded on the basis of ten randomly selected plants, while that of days to tasseling, silking and root lodging were taken on whole plot basis. Analysis of variance was carried out for all the above traits, whereas GCA and SCA were calculated following Griffing (1956) Method 2, Model II.

\section{RESULTS AND DISCUSSION}

The mean performance of 28 crosses for yield related traits along with three checks is presented in Table 1 . The presence of considerable genetic variability was obvious among traits as genotypes differed significantly. Four hybrids showed at per yield with commercial check $900 \mathrm{M}$, while the cross $\mathrm{P}_{7} \times \mathrm{Q}_{2}$ had significantly higher yield. Highly significant mean squares for GCA and SCA for all the characters clearly indicate that those traits were controlled by both types of gene actions additive and non-additive (Table 2). The results agreed with the findings of Amiruzzaman et al. (2011) and Verma and Narayan (2008) in QPM maize.

Although both additive and non-additive genetic variances are important for the traits, the higher magnitude of SCA than GCA for grain yield indicates the importance of non-additive gene action (dominance and epistasis) in its inheritance. The result is in close agreement with Bhatnagar et al. (2004), Hossain and Prasanna (2008) and Amiruzzaman et al. (2011) who reported the importance of both additive and nonadditive genetic variances with higher magnitude of SCA over GCA for yield-related characters of QPM in their study. 
Table 1. Mean performance of QPM hybrids obtained from $8 \times 8$ half diallel cross along with checks

\begin{tabular}{|c|c|c|c|c|c|c|c|c|c|c|c|}
\hline $\begin{array}{l}\text { Sl. } \\
\text { No. }\end{array}$ & $\begin{array}{r}\text { Crosses/ } \\
\text { Checks }\end{array}$ & $\begin{array}{c}\text { Days } \\
\text { to } \\
\text { tassel } \\
\end{array}$ & $\begin{array}{c}\text { Days } \\
\text { to } \\
\text { silk } \\
\end{array}$ & $\begin{array}{c}\text { Plant } \\
\text { height } \\
(\mathrm{cm})\end{array}$ & $\begin{array}{c}\text { Ear } \\
\text { height } \\
(\mathrm{cm})\end{array}$ & $\begin{array}{c}\text { Ear } \\
\text { length } \\
(\mathrm{cm})\end{array}$ & $\begin{array}{c}\text { Ear } \\
\text { diamet } \\
\text { er }(\mathrm{cm})\end{array}$ & $\begin{array}{c}\text { Grains } \\
\text { / ear } \\
\text { (no.) }\end{array}$ & $\begin{array}{c}1000 \\
\text { grain } \\
\text { wt. (g) }\end{array}$ & $\begin{array}{c}\text { Yield/ } \\
\text { plant } \\
(\mathrm{g})\end{array}$ & $\begin{array}{l}\text { Root } \\
\text { lodgin } \\
\text { g (1-5) }\end{array}$ \\
\hline 1 & $\mathrm{P}_{2} \times \mathrm{P}_{4}$ & 86 & 92 & 179 & 78 & 15 & 4 & 406 & 360 & 125.4 & 1 \\
\hline 2 & $\mathrm{P}_{2} \times \mathrm{P}_{6}$ & 94 & 99 & 190 & 99 & 14 & 4 & 349 & 360 & 126.4 & 1 \\
\hline 3 & $\mathrm{P}_{2} \times \mathrm{Q}_{3}$ & 87 & 93 & 180 & 82 & 15 & 4 & 329 & 360 & 105.6 & 1 \\
\hline 4 & $\mathrm{P}_{2} \times \mathrm{Q}_{5}$ & 92 & 98 & 198 & 95 & 16 & 4 & 448 & 380 & 141.3 & 2 \\
\hline 5 & $\mathrm{P}_{2} \times \mathrm{P}_{7}$ & 91 & 96 & 185 & 90 & 15 & 5 & 448 & 390 & 137.4 & 2 \\
\hline 6 & $\mathrm{P}_{2} \times \mathrm{Q}_{6}$ & 92 & 97 & 199 & 103 & 18 & 4 & 453 & 370 & 150.1 & 3 \\
\hline 7 & $\mathrm{P}_{2} \times \mathrm{Q}_{2}$ & 89 & 94 & 191 & 91 & 16 & 4 & 448 & 380 & 116.4 & 2 \\
\hline 8 & $\mathrm{P}_{4} \times \mathrm{P}_{6}$ & 94 & 99 & 195 & 108 & 15 & 5 & 375 & 390 & 132.7 & 2 \\
\hline 9 & $\mathrm{P}_{4} \times \mathrm{Q}_{3}$ & 86 & 92 & 181 & 92 & 17 & 4 & 434 & 395 & 136.5 & 3 \\
\hline 10 & $\mathrm{P}_{4} \times \mathrm{Q}_{5}$ & 89 & 94 & 202 & 104 & 18 & 5 & 497 & 355 & 198.0 & 2 \\
\hline 11 & $\mathrm{P}_{4} \times \mathrm{P}_{7}$ & 89 & 94 & 182 & 93 & 17 & 5 & 491 & 360 & 177.6 & 2 \\
\hline 12 & $\mathrm{P}_{4} \times \mathrm{Q}_{6}$ & 90 & 95 & 201 & 109 & 17 & 4 & 483 & 360 & 184.6 & 2 \\
\hline 13 & $\mathrm{P}_{4} \times \mathrm{Q}_{2}$ & 87 & 93 & 177 & 93 & 16 & 4 & 364 & 405 & 139.8 & 3 \\
\hline 14 & $\mathrm{P}_{6} \times \mathrm{Q}_{3}$ & 92 & 97 & 189 & 104 & 16 & 4 & 405 & 390 & 129.4 & 2 \\
\hline 15 & $\mathrm{P}_{6} \times \mathrm{Q}_{5}$ & 98 & 103 & 212 & 124 & 16 & 4 & 401 & 340 & 122.1 & 2 \\
\hline 16 & $\mathrm{P}_{6} \times \mathrm{P}_{7}$ & 94 & 99 & 196 & 111 & 16 & 4 & 448 & 430 & 153.4 & 2 \\
\hline 17 & $\mathrm{P}_{6} \times \mathrm{Q}_{6}$ & 94 & 98 & 190 & 107 & 17 & 4 & 470 & 380 & 141.0 & 3 \\
\hline 18 & $\mathrm{P}_{6} \times \mathrm{Q}_{2}$ & 93 & 97 & 192 & 102 & 16 & 4 & 378 & 340 & 164.4 & 4 \\
\hline 19 & $\mathrm{Q}_{3} \times \mathrm{Q}_{5}$ & 93 & 98 & 203 & 111 & 17 & 4 & 448 & 380 & 158.7 & 4 \\
\hline 20 & $\mathrm{Q}_{3} \times \mathrm{P}_{7}$ & 87 & 92 & 190 & 98 & 17 & 5 & 453 & 370 & 157.6 & 4 \\
\hline 21 & $\mathrm{Q}_{3} \times \mathrm{Q}_{6}$ & 88 & 93 & 196 & 101 & 18 & 4 & 490 & 370 & 167.1 & 5 \\
\hline 22 & $\mathrm{Q}_{3} \times \mathrm{Q}_{2}$ & 91 & 96 & 190 & 98 & 15 & 4 & 456 & 340 & 121.3 & 2 \\
\hline 23 & $\mathrm{Q}_{5} \times \mathrm{P}_{7}$ & 90 & 94 & 206 & 110 & 18 & 5 & 479 & 395 & 212.7 & 2 \\
\hline 24 & $\mathrm{Q}_{5} \times \mathrm{Q}_{6}$ & 100 & 105 & 173 & 97 & 14 & 4 & 338 & 330 & 93.5 & 5 \\
\hline 25 & $\mathrm{Q}_{5} \times \mathrm{Q}_{2}$ & 90 & 94 & 191 & 98 & 16 & 4 & 409 & 335 & 115.2 & 2 \\
\hline 26 & $\mathrm{P}_{7} \times \mathrm{Q}_{6}$ & 87 & 92 & 190 & 100 & 18 & 5 & 496 & 410 & 206.8 & 3 \\
\hline 27 & $\mathrm{P}_{7} \times \mathrm{Q}_{2}$ & 84 & 89 & 185 & 91 & 17 & 5 & 464 & 420 & 218.0 & 1 \\
\hline 28 & $\mathrm{Q}_{6} \times \mathrm{Q}_{2}$ & 87 & 92 & 190 & 89 & 18 & 4 & 490 & 390 & 173.5 & 1 \\
\hline 29 & BHM-5 & 89 & 94 & 193 & 106 & 17 & 4.4 & 406 & 350 & 128.5 & 5 \\
\hline 30 & BHM-7 & 85 & 90 & 197 & 108 & 17 & 4.8 & 452 & 365 & 146.2 & 4 \\
\hline 31 & $900 \mathrm{M}$ & 87 & 92 & 186 & 100 & 17.6 & 4.8 & 495 & 410 & 203.8 & 5 \\
\hline \multirow[t]{4}{*}{32} & BHM-9 & 87 & 92 & 192 & 94 & 19 & 4.1 & 470 & 380 & 166.8 & 4 \\
\hline & F-test & $* *$ & $* *$ & $* *$ & $* *$ & $* *$ & $* *$ & *** & $* *$ & $* *$ & \\
\hline & $\mathrm{CV}(\%)$ & 0.40 & 0.41 & 3.57 & 3.34 & 3.09 & 4.42 & 4.88 & 5.84 & 1.76 & \\
\hline & $\mathrm{CD}(5 \%)$ & 0.74 & 0.81 & 13.03 & 6.17 & 0.97 & 0.38 & 38.50 & 45.03 & 4.63 & \\
\hline
\end{tabular}

Scale (1-5): 1-resistant and 5-susceptible; ** Significant at 1\% level.

Table 2. Analysis of variances for combining ability of yield and yield components in QPM

\begin{tabular}{l|c|c|c|c|c|c|c|c|c|c}
\hline Source & df & $\begin{array}{c}\text { Days to } \\
\text { tassel }\end{array}$ & $\begin{array}{c}\text { Days to } \\
\text { silk }\end{array}$ & $\begin{array}{c}\text { Plant } \\
\text { height }\end{array}$ & $\begin{array}{c}\text { Ear } \\
\text { height }\end{array}$ & $\begin{array}{c}\text { Ear } \\
\text { length }\end{array}$ & $\begin{array}{c}\text { Ear } \\
\text { diameter }\end{array}$ & $\begin{array}{c}\text { Grains/ } \\
\text { ear }\end{array}$ & $\begin{array}{c}1000 \\
\text { grain } \\
\text { Wt. }\end{array}$ & $\begin{array}{c}\text { Yield/ } \\
\text { plant }\end{array}$ \\
\hline GCA & 7 & $25.5^{* *}$ & $22.1^{* *}$ & $361.2^{* *}$ & $354.3^{* *}$ & $6.5^{* *}$ & $0.2^{* *}$ & $5077^{* *}$ & $796^{* *}$ & $1396^{* *}$ \\
SCA & 28 & $24.8^{* *}$ & $22.7^{* *}$ & $465.8^{* *}$ & $262.1^{* *}$ & $3.4^{* *}$ & $0.1^{* *}$ & $9475^{* *}$ & $5720^{* *}$ & $2372^{* *}$ \\
Error & 35 & 0.07 & 0.08 & 21.0 & 4.7 & 0.1 & 0.02 & 183.4 & 390.8 & 2.6 \\
\hline$\sigma^{2} \mathrm{GCA} / \sigma^{2} \mathrm{SCA}$ & & 0.003 & 0.003 & 0.02 & 0.03 & 0.09 & 0.14 & 0.04 & 0.09 & 0.04 \\
$\sigma_{\mathrm{A}}^{2}$ & 0.13 & 0.12 & 20.92 & 18.42 & 0.63 & 0.03 & 879.61 & 984.83 & 195 \\
$\sigma_{\mathrm{D}}^{2}$ & 24.78 & 22.66 & 444.83 & 257.47 & 3.28 & 0.11 & 9291.42 & 5329.59 & 2369 \\
\hline$*$ Significant at 1\% level.
\end{tabular}

\section{General Combining Ability (GCA) Effects}

The GCA effects of parents were calculated and have been presented in Table 3. based on their effects parents were grouped as good, average and poor general combiners. Parents having desirable and significant GCA effect were considered as good general 
combiners, whereas average parents as average combiners. Parents possessed significant but negative or undesirable GCA effects were designated as poor or low combiners.

Table 3. General combining ability (GCA) effects and mean performance (in parenthesis) of parents for yield and yield related characters in QPM

\begin{tabular}{|c|c|c|c|c|c|c|c|c|c|c|}
\hline $\begin{array}{l}\text { Sl. } \\
\text { No. }\end{array}$ & Parents & $\begin{array}{l}\text { Days } \\
\text { to } \\
\text { tassel }\end{array}$ & $\begin{array}{c}\text { Days to } \\
\text { silk }\end{array}$ & $\begin{array}{l}\text { Plant } \\
\text { height }\end{array}$ & $\begin{array}{c}\text { Ear } \\
\text { height }\end{array}$ & $\begin{array}{c}\text { Ear } \\
\text { length }\end{array}$ & $\begin{array}{c}\text { Ear } \\
\text { diameter }\end{array}$ & $\begin{array}{c}\text { Grains/ } \\
\text { ear }\end{array}$ & $\begin{array}{l}1000 \\
\text { grain } \\
\text { Wt. }\end{array}$ & $\begin{array}{l}\text { Yield/ } \\
\text { plant }\end{array}$ \\
\hline 1 & $\mathrm{P}_{2}$ & $\begin{array}{c}0.1 \\
(101)\end{array}$ & $\begin{array}{l}0.4 * * \\
(105)\end{array}$ & $\begin{array}{l}-7.6 * * \\
(120)\end{array}$ & $\begin{array}{c}-8.7 * * \\
(52)\end{array}$ & $\begin{array}{c}-0.8 * * \\
(12)\end{array}$ & $\begin{array}{l}-0.1 \\
(3.8)\end{array}$ & $\begin{array}{c}-16.8 * * \\
(240)\end{array}$ & $\begin{array}{c}-5.4 \\
(225)\end{array}$ & $\begin{array}{c}-14.2 * * \\
(67.5)\end{array}$ \\
\hline 2 & $\mathrm{P}_{4}$ & $\begin{array}{c}-1.3 * * \\
(99)\end{array}$ & $\begin{array}{c}-1.1 * * \\
(103)\end{array}$ & $\begin{array}{l}-6.5 * * \\
(127)\end{array}$ & $\begin{array}{c}-4.5^{* * *} \\
(54)\end{array}$ & $\begin{array}{c}0.1 \\
(13)\end{array}$ & $\begin{array}{c}0.2 * * \\
(4.5\end{array}$ & $\begin{array}{c}-4.0 \\
(220)\end{array}$ & $3.1(260)$ & $\begin{array}{l}4.4 * * \\
(64.5)\end{array}$ \\
\hline 3 & $\mathrm{P}_{6}$ & $\begin{array}{l}2.7 * * \\
(100)\end{array}$ & $\begin{array}{l}2.5^{* *} \\
(104)\end{array}$ & $\begin{array}{l}4.2 * * \\
(157)\end{array}$ & $\begin{array}{c}6.7^{* * *} \\
(70)\end{array}$ & $\begin{array}{c}-0.9 * * \\
(10)\end{array}$ & $\begin{array}{c}-0.1 \\
(3.8)\end{array}$ & $\begin{array}{c}-40.3^{* *} \\
(160)\end{array}$ & $\begin{array}{c}-1.4 \\
(240)\end{array}$ & $\begin{array}{c}-11.1 * * \\
(49.5)\end{array}$ \\
\hline 4 & $\mathrm{Q}_{3}$ & $\begin{array}{c}-1.6^{* * *} \\
(96)\end{array}$ & $\begin{array}{c}-1.5 * * \\
(100)\end{array}$ & $\begin{array}{c}-1.1 \\
(149)\end{array}$ & $\begin{array}{c}-2.8^{* * *} \\
(57)\end{array}$ & $\begin{array}{c}0.2 \\
(14)\end{array}$ & $\begin{array}{l}-0.1 \\
(4.1)\end{array}$ & $\begin{array}{l}10.3^{*} \\
(299)\end{array}$ & $\begin{array}{l}-1.4 \\
(242)\end{array}$ & $\begin{array}{l}-5.6 * * \\
(73.5)\end{array}$ \\
\hline 5 & $\mathrm{Q}_{5}$ & $\begin{array}{c}1.8^{* *} \\
(99)\end{array}$ & $\begin{array}{l}1.6^{* *} \\
(104)\end{array}$ & $\begin{array}{c}10.8 * * \\
(180)\end{array}$ & $\begin{array}{c}8.8^{* * *} \\
(89)\end{array}$ & $\begin{array}{c}0.1 \\
(14)\end{array}$ & $\begin{array}{l}-0.1 \\
(3.8)\end{array}$ & $0.3(256)$ & $\begin{array}{l}-10.4 \\
(240)\end{array}$ & $\begin{array}{c}-0.3 \\
(67.5)\end{array}$ \\
\hline 6 & $\mathrm{P}_{7}$ & $\begin{array}{c}-1.0 * * \\
(100)\end{array}$ & $\begin{array}{c}-1.0 * * \\
(105\end{array}$ & $\begin{array}{c}-0.5 \\
(149)\end{array}$ & $1.1(74)$ & $\begin{array}{l}1.5^{* *} \\
(18)\end{array}$ & $\begin{array}{l}0.3 * * \\
(4.4)\end{array}$ & $\begin{array}{c}32.7^{* *} \\
(289)\end{array}$ & $\begin{array}{l}18.6 * * \\
(272)\end{array}$ & $\begin{array}{l}22.2 * * \\
(69.0)\end{array}$ \\
\hline 7 & $\mathrm{Q}_{6}$ & $\begin{array}{c}0.3^{* *} * \\
(99)\end{array}$ & $\begin{array}{l}0.2 * * \\
(103)\end{array}$ & $\begin{array}{l}3.1 * \\
(164)\end{array}$ & $\begin{array}{c}2.9 * * \\
(76)\end{array}$ & $\begin{array}{c}0.6^{* * *} \\
(14)\end{array}$ & $\begin{array}{l}-0.1 \\
(4.1)\end{array}$ & $\begin{array}{l}21.0^{* * *} \\
(260)\end{array}$ & $3.6(260)$ & $\begin{array}{l}9.3 * * \\
(78.0)\end{array}$ \\
\hline 8 & $\mathrm{Q}_{2}$ & $\begin{array}{c}-1.1 * * \\
(99)\end{array}$ & $\begin{array}{c}-1.1^{* *} \\
(104)\end{array}$ & $\begin{array}{c}-2.6 \\
(148)\end{array}$ & $\begin{array}{c}-3.4 * * \\
(66)\end{array}$ & $\begin{array}{c}-0.7 * * \\
(10)\end{array}$ & $\begin{array}{c}-0.3 * * \\
(2.6)\end{array}$ & $\begin{array}{l}-3.1 \\
(245) \\
\end{array}$ & $\begin{array}{c}-6.9 \\
(250) \\
\end{array}$ & $\begin{array}{c}-4.7 * * \\
(42.0)\end{array}$ \\
\hline & $\begin{array}{l}\mathrm{SE}(\mathrm{gi}) \\
\mathrm{LSD}_{(5 \%)}\end{array}$ & $\begin{array}{l}0.08 \\
0.18\end{array}$ & $\begin{array}{l}0.08 \\
0.19\end{array}$ & $\begin{array}{l}1.36 \\
3.20\end{array}$ & $\begin{array}{l}0.64 \\
1.51\end{array}$ & $\begin{array}{l}0.10 \\
0.22\end{array}$ & $\begin{array}{l}0.04 \\
0.09\end{array}$ & $\begin{array}{l}4.01 \\
9.47\end{array}$ & $\begin{array}{c}5.85 \\
13.82\end{array}$ & $\begin{array}{l}0.48 \\
1.12\end{array}$ \\
\hline
\end{tabular}

$*$ and $* *$ Significant at $5 \%$ and $1 \%$ level. Values in parenthesis are mean of the traits.

To develop early and short stature hybrid(s), which would not be laid down by strong wind, hail and/or storm, then negative significant GCA effects for days to tasseling, silking, plant height and ear height are to be considered. From the Table 3, it was observed that parents $\mathrm{P}_{4}, \mathrm{P}_{7}, \mathrm{Q}_{2}$ and $\mathrm{Q}_{3}$ for both days to tassel and silk, $\mathrm{P}_{2}$ and $\mathrm{P}_{4}$ for plant height and ear height exhibited significant negative GCA effects. The good general combiners for major yield determining characters were $\mathrm{P}_{7}$ and $\mathrm{Q}_{6}$ (longer ears); $\mathrm{P}_{4}$ and $\mathrm{P}_{7}$ (thick ears); $\mathrm{P}_{7}, \mathrm{Q}_{3}$ and $\mathrm{Q}_{6}$ (higher number of kernels per ear) and $\mathrm{P}_{7}$ (bold kernels). Positive estimates for these traits are desirable since these traits directly contribute to yield in maize.

Parents $\mathrm{P}_{4}, \mathrm{P}_{7}$ and $\mathrm{Q}_{6}$ were good combiner of yield and some important yield contributing traits (Table 3). These parents could be used in breeding program for obtaining higher yield and some of the desirable traits. Parent $\mathrm{P}_{7}$ was the best general combiner for yield. It had significant positive GCA value for all yield components along with high mean indicated the parent could be very useful for combining more positive alleles. The result is confirmed by the findings of Amiruzzamam et al. (2010), Ivy and hawlader (2000) and Hussain et al. (2003). Finally, it can suggest that $\mathrm{P}_{7}$ was an excellent combiner of yield and yield contributing traits and could be used extensively in hybrid breeding program with a view to increase yield.

\section{Specific Combining Ability (SCA) Effects}

The SCA effects of the crosses for all the characters studied are presented in Table 4 . The desirable significant negative SCA effect was obtained from 22 crosses for both days to tasseling and silking. Cross $\mathrm{Q}_{5} \times \mathrm{Q}_{6}$ had significant but negative SCA effect for both plant and ear height, while $\mathrm{Q}_{6} \times \mathrm{Q}_{2}$ showed for only ear height. Significant positive SCA effects were desirable for ear length, ear diameter, number of grains per ear and 
1000-grain weight and different numbers of cross combinations were identified as the best combinations for those characters (Table 4).

Among the crosses, twenty three had significant positive SCA effects for yield per plant and in most of the cases, one or both parents were good combiners, which indicates the vital role of parental lines' GCA. Xingming et al. (2002) also reported similar result. The crosses with high SCA value also had high per se performance (Table 1). Vasal (1998) revealed that enrollment of at least one good combiner in crossing program prioritize higher heterosis in maize which confirmed the findings of the present study. To obtain heterosis for a complex trait like yield, a superior parent for one component should be crossed with a parent superior for another. Vasal (1998) also suggested that both combining ability and per se performance are important and thus balanced resources must be spent on each of these two aspects. The highest value of SCA effects for yield was observed in $\mathrm{P}_{7} \times \mathrm{Q}_{2}(69.4)$ followed by $\mathrm{P}_{4} \times \mathrm{Q}_{5}(62.8)$ and $\mathrm{Q}_{5} \times$ $\mathrm{P}_{7}$ (59.7). The crosses involved high $\times$ high, average $\times$ average, low $\times$ low, high $\times$ average or high $\times$ low general combining parents yielded in desirable significant SCA effects for different characters, manifested attribution of sizeable additive $\times$ additive gene action of good combiner parents.

Table 4. Specific combining ability (SCA) effects for yield and yield related characters in $8 \times 8$ diallel cross of QPM hybrids

\begin{tabular}{|c|c|c|c|c|c|c|c|c|c|}
\hline Crosses & $\begin{array}{c}\text { Days to } \\
\text { tassel }\end{array}$ & $\begin{array}{c}\text { Days to } \\
\text { silk }\end{array}$ & $\begin{array}{c}\text { Plant } \\
\text { height }\end{array}$ & $\begin{array}{c}\text { Ear } \\
\text { height }\end{array}$ & $\begin{array}{c}\text { Ear } \\
\text { length }\end{array}$ & $\begin{array}{c}\text { Ear } \\
\text { diameter }\end{array}$ & $\begin{array}{c}\text { Grains/ } \\
\text { ear }\end{array}$ & $\begin{array}{c}1000 \\
\text { grain Wt. }\end{array}$ & $\begin{array}{l}\text { Yield/ } \\
\text { plant }\end{array}$ \\
\hline $\mathrm{P}_{2} \times \mathrm{P}_{4}$ & $-5.2 * *$ & $-4.5^{* *}$ & $11.3^{* *}$ & -1.2 & 0.1 & 0.1 & $34.7 * *$ & $24.1 * *$ & $4.1 * *$ \\
\hline $\mathrm{P}_{2} \times \mathrm{P}_{6}$ & $-1.2^{* *}$ & $-1.2^{* *} *$ & $11.5^{* *}$ & $8.5^{* *}$ & $0.6 * *$ & -0.1 & -5.9 & $28.6 * *$ & $20.7^{* *} *$ \\
\hline $\mathrm{P}_{2} \times \mathrm{Q}_{3}$ & $-3.8^{* *}$ & $-3.1 * *$ & $6.4^{* *}$ & 1.5 & $-0.4^{* *}$ & $-0.3^{* *}$ & $-36.6 * *$ & $28.6 * *$ & $-5.6^{* *}$ \\
\hline $\mathrm{P}_{2} \times \mathrm{Q}_{5}$ & $-2.3^{* *}$ & $-1.8^{* *}$ & $12.4 * *$ & $2.9^{* *}$ & $0.6 * *$ & $0.2 * *$ & $72.4^{* *}$ & $57.6 * *$ & $24.7^{* *} *$ \\
\hline $\mathrm{P}_{2} \times \mathrm{P}_{7}$ & $-0.5^{* *}$ & $-0.6^{* *}$ & $10.7 * *$ & $5.1 * *$ & $-1.1^{* *}$ & $0.2^{*}$ & $39.9 * *$ & $38.6 * *$ & $-1.7 *$ \\
\hline $\mathrm{P}_{2} \times \mathrm{Q}_{6}$ & $-1.3^{* *}$ & $-1.5^{* *}$ & $21.6^{* *}$ & $16.3^{* *}$ & $2.3 * *$ & $0.3^{* *}$ & $56.7 * *$ & $33.6 * *$ & $23.9^{* *}$ \\
\hline $\mathrm{P}_{2} \times \mathrm{Q}_{2}$ & $-2.3^{* *} *$ & $-2.5 * *$ & $19.3^{* *}$ & $11.1^{* *}$ & $1.9 * *$ & $0.3^{* *}$ & $75.7 * *$ & $54.1 * *$ & $4.2 * *$ \\
\hline $\mathrm{P}_{4} \times \mathrm{P}_{6}$ & $0.2 *$ & $0.4 * *$ & $15.5 * *$ & $13.8 * *$ & 0.1 & $0.2 * *$ & $27.2 * *$ & $50.1 * *$ & $8.4 * *$ \\
\hline $\mathrm{P}_{4} \times \mathrm{Q}_{3}$ & $-3.9 * *$ & $-3.0 * *$ & $6.3 * *$ & $6.9 * *$ & $1.5^{* *}$ & -0.1 & $35.5^{* *}$ & $55.1 * *$ & $6.6^{* *}$ \\
\hline $\mathrm{P}_{4} \times \mathrm{Q}_{5}$ & $-3.9 * *$ & $-3.7 * *$ & $15.9 * *$ & $7.2 * *$ & $2.6^{* *}$ & $0.2 * *$ & $108.6^{* *}$ & $29.1 * *$ & $62.8^{* *}$ \\
\hline $\mathrm{P}_{4} \times \mathrm{P}_{7}$ & $-1.0^{* *}$ & $-1.1 * *$ & $7.2 * *$ & $3.9^{* *}$ & 0.3 & $-0.2 * *$ & $70.1 * *$ & 0.1 & $19.9^{* *}$ \\
\hline $\mathrm{P}_{4} \times \mathrm{Q}_{6}$ & $-1.9^{* *}$ & $-1.8^{* *}$ & $22.6^{* *}$ & $18.6^{* *}$ & $0.3^{*}$ & $-0.1^{* *}$ & $73.3^{* *}$ & 15.1 & $39.9^{* *}$ \\
\hline $\mathrm{P}_{4} \times \mathrm{Q}_{2}$ & $-2.9^{* *}$ & $-2.4^{* *}$ & $3.8^{*}$ & $8.4 * *$ & $0.7 * *$ & 0.1 & $-21.1 * *$ & $55.6 * *$ & $9.0 * *$ \\
\hline $\mathrm{P}_{6} \times \mathrm{Q}_{3}$ & $-1.4 * *$ & $-1.2 * *$ & 3.6 & $7.6 * *$ & $1.4 * *$ & $0.2 * *$ & $42.9 * *$ & $54.5^{* *}$ & $15.1^{* *}$ \\
\hline $\mathrm{P}_{6} \times \mathrm{Q}_{5}$ & $1.1 * *$ & $1.1 * *$ & $15.1 * *$ & $16.5 * *$ & $0.8 * *$ & -0.1 & $48.9 * *$ & 13.6 & $2.4 * *$ \\
\hline $\mathrm{P}_{6} \times \mathrm{P}_{7}$ & -0.1 & $0.2^{*}$ & $10.4 * *$ & $11.2 * *$ & 0.1 & $-0.1^{*}$ & $63.5^{* *}$ & $74.6 * *$ & $11.3 * *$ \\
\hline $\mathrm{P}_{6} \times \mathrm{Q}_{6}$ & $-1.4 * *$ & $-2.0 * *$ & 0.8 & $5.4 * *$ & $1.7 * *$ & $0.1^{*}$ & $97.2 * *$ & $39.6 * *$ & $11.7^{* * *}$ \\
\hline $\mathrm{P}_{6} \times \mathrm{Q}_{2}$ & $-1.4^{* *}$ & $-1.6^{* *}$ & $8.5^{* *}$ & $6.7 * *$ & $1.6^{* *}$ & $0.4^{* *}$ & $29.2 * *$ & -9.9 & $49.1^{* *}$ \\
\hline $\mathrm{Q}_{3} \times \mathrm{Q}_{5}$ & -0.1 & 0.2 & $10.9^{* *}$ & $13.1 * *$ & $0.6^{* *}$ & $0.1^{* *}$ & $45.3 * *$ & $53.6 * *$ & $33.6^{* *}$ \\
\hline $\mathrm{Q}_{3} \times \mathrm{P}_{7}$ & $-3.2 * *$ & $-3.2 * *$ & $9.8^{* *}$ & $7.7 * *$ & -0.1 & 0.1 & $17.3 * *$ & 14.6 & 10.0 ** \\
\hline $\mathrm{Q}_{3} \times \mathrm{Q}_{6}$ & $-3.0^{* *}$ & $-2.9^{* * *}$ & $11.7 * *$ & $8.4 * *$ & $1.2 * *$ & $-0.2^{* *}$ & $66.5 * *$ & $29.6 * *$ & $32.3 * *$ \\
\hline $\mathrm{Q}_{3} \times \mathrm{Q}_{2}$ & $1.5 * *$ & $1.5^{* *}$ & $11.4^{* *}$ & $12.2 * *$ & $-0.4^{* *} *$ & $0.2 * *$ & $56.6 * *$ & 10.1 & 0.6 \\
\hline $\mathrm{Q}_{5} \times \mathrm{P}_{7}$ & $-3.7 * *$ & $-4.4 * *$ & $13.8 * *$ & $7.6 * *$ & $1.0 * *$ & $0.4 * *$ & $53.9 * *$ & $48.5^{* *}$ & $59.7 * *$ \\
\hline $\mathrm{Q}_{5} \times \mathrm{Q}_{6}$ & & & & & & & & & - \\
\hline \multirow{3}{*}{$\mathrm{Q}_{5} \times \mathrm{Q}_{2}$} & $4.9 * *$ & $5.4 * *$ & $-22.8^{*}$ & $-6.7 * *$ & $-2.8^{* *} *$ & $-0.5 * *$ & $-75.4 *$ & -1.4 & $46.6^{* * *}$ \\
\hline & & & & & & & & & - \\
\hline & $-3.0 * *$ & $-3.7 * *$ & 0.4 & 0.6 & $0.9 * *$ & $0.2 * *$ & $19.6 * *$ & 14.1 & $10.8^{* * *}$ \\
\hline $\mathrm{P}_{7} \times \mathrm{Q}_{6}$ & $-4.7 * *$ & $-4.5^{* *}$ & $5.5^{* *}$ & $3.5 * *$ & $0.4 * *$ & $0.3^{* *}$ & $50.1 * *$ & $59.6 * *$ & $44.3 * *$ \\
\hline $\mathrm{P}_{7} \times \mathrm{Q}_{2}$ & $-6.2^{* *}$ & $-6.1^{* *}$ & $6.2 * *$ & 1.3 & $0.3^{*}$ & $0.5^{* *}$ & $42.2 * *$ & $30.1 * *$ & $69.4 * *$ \\
\hline $\mathrm{Q}_{6} \times \mathrm{Q}_{2}$ & $-4.5^{* *}$ & $-4.3 * *$ & $7.6^{* *}$ & $-3.0 * *$ & $2.4^{* *}$ & $0.4 * *$ & $79.9 * *$ & $55.1 * *$ & $37.8^{* * *}$ \\
\hline SE(ij) & 0.33 & 0.36 & 5.79 & 2.74 & 0.40 & 0.18 & 17.13 & 25.00 & 2.06 \\
\hline $\operatorname{LSD}_{(5 \%)}$ & 0.67 & 0.73 & 11.87 & 5.62 & 0.82 & 0.37 & 35.08 & 51.21 & 4.22 \\
\hline
\end{tabular}

* Significant at 5 percent level; ** Significant at 1 percent level 
Among the higher three SCA effects $\mathrm{P}_{7} \times \mathrm{Q}_{2}$ belonged to high $\times$ low combination, manifesting complementary gene action as well as additive effect of the high parent, while $\mathrm{P}_{4} \times \mathrm{Q}_{5}$ and $\mathrm{Q}_{5} \times \mathrm{P}_{7}$ belonged to high $\times$ average combinations. The low $\times$ low cross combinations with appreciable SCA effects might be ascribed as non-allelic gene action of dominance $\times$ dominance type which produced over dominance. So, the superior performance of most hybrids might be the result of

epistatic interaction and the GCA effects of their parents were not reflected in SCA. This result is in accordance with the findings of Ivy and Howlader (2000). Moreover, Amiruzzaman et al. (2011) also pointed out that the SCA is a result of the interaction of GCA effects of the parents and that it can improve or deteriorate the hybrid expression compared to the expected effect based on GCA only.

Considering the mean performance and significant desirable SCA effects three crosses $\mathrm{Q}_{5} \times \mathrm{P}_{7}, \mathrm{P}_{7} \times \mathrm{Q}_{6}$ and $\mathrm{P}_{7} \times \mathrm{Q}_{2}$ could be used for commercial variety development after verifying their performance over locations, and parents $\mathrm{P}_{4}, \mathrm{P}_{7}$ and $\mathrm{Q}_{6}$ could be utilized in different breeding program.

\section{References}

Amiruzzaman, M., M.A. Islam, L. Hasan, M. Kadir and M.M. Rohman. 2011. Heterosis and combining ability in a diallel among elite inbred lines of maize (Zea mays L.). Emir. J. Agril. 23: 204-208.

Amiruzzaman, M., M.A. Islam, L. Hassan and M.M. Rohman. 2010. Combining ability and heterosis for yield and component characters in maize. Aca. J. Pl. Sci. 3(2): 79-84.

Baral, B. 2016. Agriculture Market Information System (AMIS) in Bangladesh. $26^{\text {th }}$ Session on Asia and Pacific Commission on Agriculture Statistics, Thimphu, Bhutan. Feb. 15-19:3

Barata C., Carena M. 2006. Classification of North Dakota maize inbred lines into heterotic groups based on molecular and testcross data. Euphytica, 151: 339349.

Bello O.B., Olaoye G. 2009. Combining ability for maize grain yield and other agronomic characters in a typical southern guinea savanna ecology of Nigeria. Afr. J. Biotechnol. 11: 2518-2522.

Bhatnagar, S., E.J. Bertran and L.W. Rooney. 2004. Combining abilities of quality protein maize inbreds. Crop Sci. 44: 1997-2005.

Bodker, L., E. Wulff, and J. Thorp. 2006. Seed sector country profile: Bangladesh H. Volume I: overview of seed supply systems and seed health issues. Copenhagen: Danish seed health centre for developing countries.

Fan X.M., Tan J., Yang J.Y., Liu F., Huang B.H., Huang Y.X. 2002. Study on combining ability for yield and genetic relationship between exotic tropical, subtropical maize inbreeds and domestic temperate maize inbreeds. Sci. Agric. Sinica, 35: 743-749.

FAO. 2012. FAOSTAT, Production. Cited February 12, 2014. http://faostat.fao.org/site/567/ DesktopDefault.aspx. PageID=567\#ancor.

Griffing, B. 1956. Concept of general and specific combining ability in relation to diallel crossing system. Aus. J. Bio. Sci. 9: 463-493.

Harun-Ar-Rashid, M. Ali and D. Gisselquist. 2012. Private-Sector Agricultural Research and Innovation in Bangladesh Overview, Impact and Policy Options. International Food Policy Research Institute, Bangladesh. Pp: 15 
Hossain, F. and B. M. Prasanna. 2008. Genetic and biochemical analysis of quality protein maize (QPM) lines in India. Book of Abstracts. The 10th Asian Regional Maize Workshop. Indonesia, Oct. 20-23. P: 7.

Hussain, S.A., M. Amiruzzaman and Z. Hossain. 2003. Combining ability estimates in maize. Bangladesh J. Agril. Res. 28: 435-440.

Ivy, N.A. and M.S. Howlader. 2000. Combining ability in maize. Bangladesh J. Agril. Res. 25: 385-392.

Koutsika-Sotiriou, M. 1999. Hybrid seed production in maize. In Basra AS (Ed.), Heterosis and hybrid seed production in agronomic crops. Food Products Press, New York. Pp: 25-64.

Melani M.D., Carena M.J. 2005. Alternative maize heterotic pattern for the Northern Corn Belt. Crop Sci. 45: 2186-2194.

Pavan, R. 2009. Gene action and combining ability studies in single cross hybrids of maize (Zea mays L.). M.S. Dissertation. Department of genetics and plant breeding. University of Agricultural Sciences. Dharwad. P: 1

Vasal, S.K. 1998. Hybrid maize technology: Challenges and expanding possibilities for research in the next century. In: Vasal, S.K., C.F. Gonzalez and F. Xingming (ed). Proc. $7^{\text {th }}$ Asian Reg. Maize Workshop. Philippines, Feb. 23-27: 58-62.

Verma, S.S. and A. Narayan. 2008. Heterosis, combining ability and phenotypic stability for yield and other characters in high quality protein maize (Zea mays L.). Book of Abstracts. The 10th Asian Reg. Maize Workshop. Indonesia, Oct. 20-23. P: 89.

Xingming, F., J. Tan, Z. Chen and J. Yang. 2002. Combining ability and heterotic grouping of ten temperate, tropical and subtropical quality protein maize. In: Srinivasan, G., P.H. Zaidi, B.N. Prasanna, F.C. Gonzalez and K. Lesnick (ed). Proc. 8th Asian Reg. Maize Workshop. Thailand. Aug. 5-8: 10-18. 